\title{
Modeling the transmission and thermal emission in a pupil image behind the Keck II adaptive optics system
}

\author{
Pauline Arriaga ${ }^{a}$, Michael P. Fitzgerald ${ }^{\mathrm{a}}$, James E. Lyke ${ }^{\mathrm{b}}$, Randall D. Campbell ${ }^{\mathrm{b}}$, Peter L. \\ Wizinowich $^{\mathrm{b}}$, Sean M. Adkins ${ }^{\mathrm{b}}$, and Keith Y. Matthews ${ }^{\mathrm{c}}$ \\ aDepartment of Physics \& Astronomy, University of California, Los Angeles, CA 90095, USA \\ ${ }^{\mathrm{b}}$ W. M. Keck Observatory, HI, USA \\ ${ }^{\mathrm{c}}$ Caltech Optical Observatories, California Institute of Technology, Pasadena, CA, USA
}

\begin{abstract}
The design and performance of astronomical instruments depend critically on the total system throughput as well as the background emission from the sky and instrumental sources. In designing a pupil stop for backgroundlimited imaging, one seeks to balance throughput and background rejection to optimize measurement signal-tonoise ratios. Many sources affect transmission and emission in infrared imaging behind the Keck Observatorys adaptive optics systems, such as telescope segments, segment gaps, secondary support structure, and AO bench optics. Here we describe an experiment, using the pupil-viewing mode of NIRC2, to image the pupil plane as a function of wavelength. We are developing an empirical model of throughput and background emission as a function of position in the pupil plane. This model will be used in part to inform the optimal design of cold pupils in future instruments, such as the new imaging camera for OSIRIS.
\end{abstract}

Keywords:

\section{INTRODUCTION}

The function of a pupil stop is to block unwanted scattered light and thermal instrumental background while allowing as much of the signal's light through. In a perfect system, the shape of the optimal pupil mask would be matched to the shape of the primary mirror, secondary obscuration, and secondary support structures. However, given physical manufacturing limits, pupil nutation and rotation, and alignment errors, it is sometimes not favorable to use such a well-matched geometry. In this work, we will use images from the pupil imaging mode of NIRC2 to motivate the design choices for future pupil mask construction on Keck II. We have constructed a model of the various components and their respective fluxes. We have then used those images to test hexagonal masks, to be used in the pupil tracking "vertical angle" mode, and annular masks to be used in the sky tracking "position angle" mode.

\section{DATA}

We took images of the Keck II pupil using NIRC2's pupil viewing mode in which a pupil imaging lens is inserted to the beam via the instrument's slide mechanism. The images were taken in $\mathrm{Kp}, \mathrm{PAH}$ and $\mathrm{Br}-\alpha$ with integration times of 60,1 , and .18 seconds, respectively. The pupil mode only obtains a partial image of the pupil. The grism slide was translated between groups of exposures for a larger effective field of view. We took three images at each patch in each band at twilight for a total of 18 images. The sky was observed in position angle mode so the images have a slight rotation between the images. Each of the images were flat field corrected, though still exhibit some vignetting inherent to the pupil viewing mode. We did not correct for bad pixels, electing instead to exclude them from subsequent analysis. A mosaic of all 6 images in $\mathrm{Kp}$ band is shown in Figure 1a. The images have a limited width and have a maximum outer radius. Regions outside of this maximum outer radius were excluded.

Further author information: (Send correspondence to P.A.)

P.A.: E-mail: parriaga@astro.ucla.edu

Ground-based and Airborne Instrumentation for Astronomy VI, edited by Christopher J. Evans, Luc Simard, Hideki Takami Proc. of SPIE Vol. 9908, 990835 · ( 2016 SPIE · CCC code: 0277-786X/16/\$18 · doi: 10.1117/12.2233068 


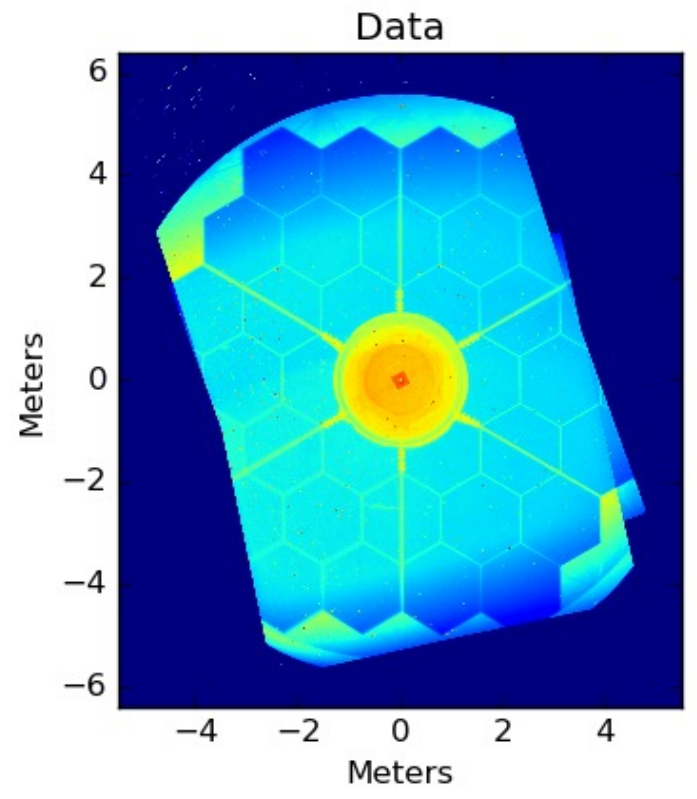

(a) Mosaic of the six images taken in Kp band.

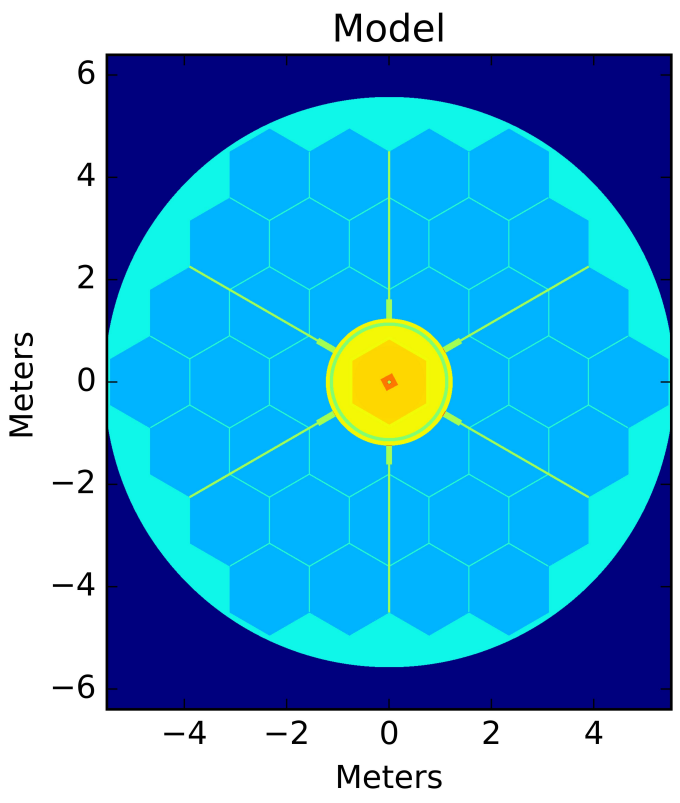

(b) Model fit of the Kp image

Figure 1

\section{MODEL}

The various components in the plane of the primary mirror are shown in Figure 2a while the components in the plane of the secondary mirror are shown in Figure 2b. The sizes of the components were either assigned from known values or measured from the data (Le Mignant et al. 1996, Nelson et al 1985). We constructed the model images by summing the fluxes of each of the emitting components. Additionally, there was a uniform thermal background component added everywhere.

We then fit our model image to the six images in each band simultaneously by generating our model at the sampling, rotation and orientations of each data image. We masked out portions of the image which were most affected by vignetting and Gaussian blurred the images to compensate for the slight field distortion at the edges of the image. We then fit for each of the model component fluxes at each wavelength using a linear least squares algorithm.

\section{RESULTS}

\subsection{Model Fit}

The resultant image fit in the Kp band is shown in Figure 1b, and is well matched to the data image shown in Figure 1a. The fits in other bands are similarly well fit. There were a number of degeneracies in the flux parameters which prevented us from obtaining physical fluxes of the various components. Firstly, all extended components such as the dome, secondary obscuration and sky were completely degenerate with the uniform background that arises from emission occuring far from the pupil plane — increasing the uniform background parameter decreased the flux parameters of all of the extended components. Secondly, the sky flux parameter was highly degenerate with the flux parameter of the primary mirror. The portion of the primary mirror which is not illuminated by the sky due to the secondary obscuration, shown in yellow in Figure 2a, is very small and overlaps with many of the components in the secondary plane. Qualitative measurements of the mirror flux show it to be less than $20 \mathrm{ADU} /$ pixel — on order of the image noise.

Though we cannot currently convert our flux parameters into physical units, we can generally characterize the emission. In Figure 3 we show the fits for the longest wavelength filter, Br- $\alpha$, and shortest wavelengths filter, Kp. Overall, the flux values of $\mathrm{Br}-\alpha$ in units of electrons $/ \mathrm{s} / \mu \mathrm{m} / \mathrm{pixel}$ are much larger as the thermal components emit 


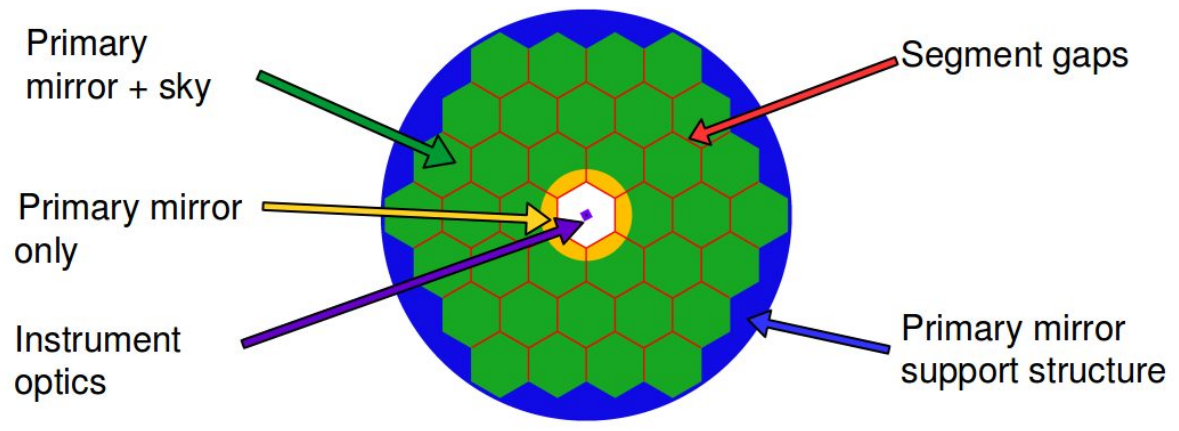

(a) Model components in the secondary plane. In blue, there is some thermal background from the dome and support structure of the primary mirror. The green component is a combination of the primary mirror's thermal emission and the reflected sky emission. In the yellow portion, sky flux is blocked by the secondary obscuration, so is comprised only of the primary mirror's emission. The purple box is some component in the instrument optics reflected back by the secondary mirror

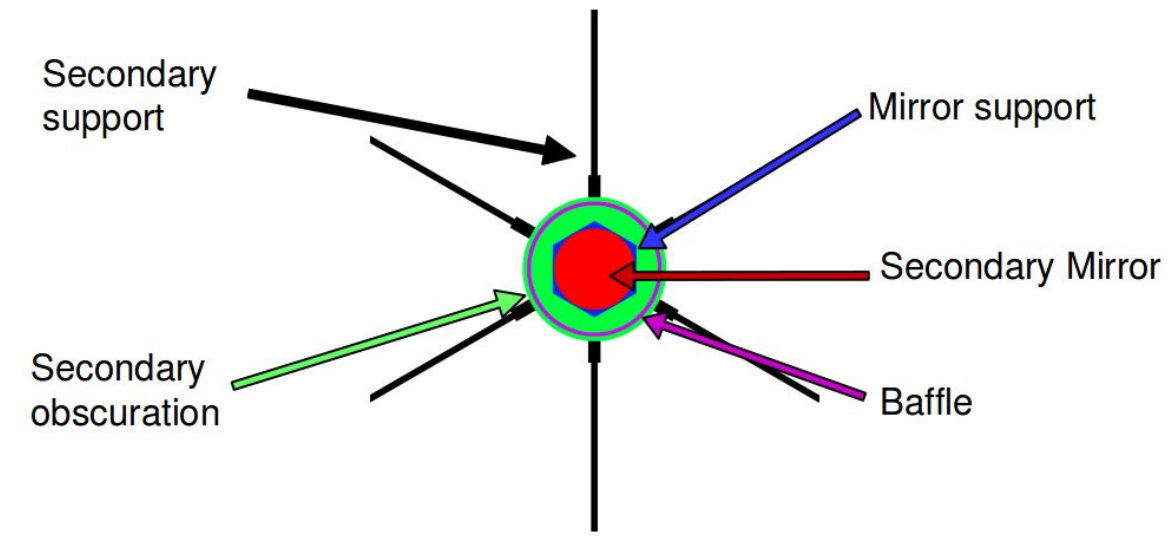

(b) Components in the plane of the secondary mirror. The secondary support holds the secondary mirror scaffold shown in light green. In front of this scaffold are the baffle tube in purple, other secondary mirror scaffolding and the secondary mirror itself which are completely opaque to the emission of the secondary obscuration.

Figure 2: Model components. In each band each component was assigned a value and added into the images. Flux in the primary plane was obscured by components in the secondary plane unless it is reflected by the secondary mirror 


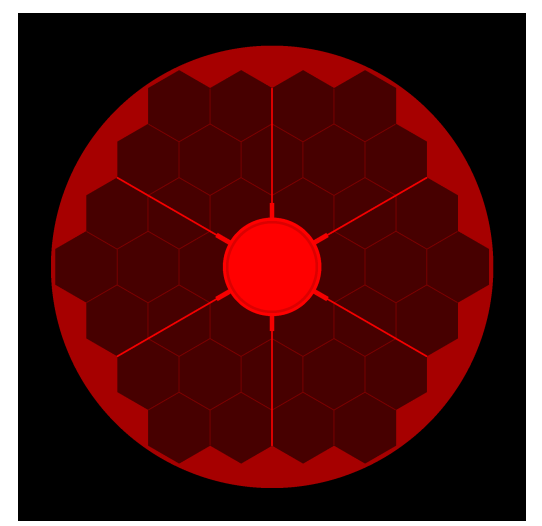

(a) Image fit for $\operatorname{Br}-\alpha$.

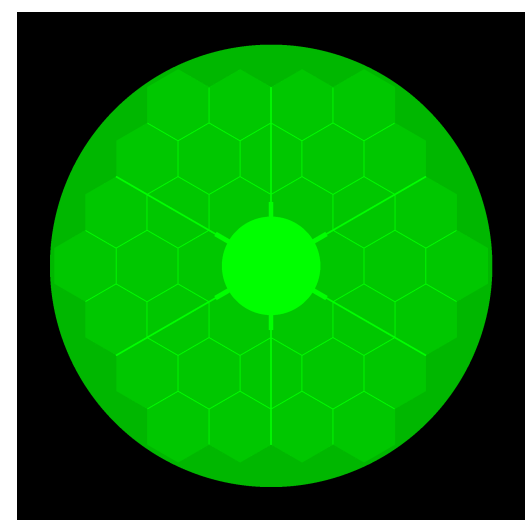

(b) Image fit for $\mathrm{Kp}$

Figure 3: Image fits of $\operatorname{Br}-\alpha(4.1 \mu \mathrm{m})$ and $\mathrm{Kp}(2.1 \mu \mathrm{m})$. The uniform background is much more prominent in $\mathrm{Kp}$, while the thermal emission is more dominant in $\operatorname{Br}-\alpha$

\begin{tabular}{|c|c|c|c|c|}
\hline & \multicolumn{2}{|c|}{ Br- $\alpha$} & \multicolumn{2}{c|}{ Kp } \\
\hline Mask & Radius (m) & Relative SNR & Radius (m) & Relative SNR \\
\hline Outer hexagon & 5.4 & 1.06 & 5.4 & 1.039 \\
Outer annulus & 4.88 & 1.11 & 4.92 & 1.072 \\
Inner hexagon & 1.39 & 1.06 & 1.38 & 1.046 \\
Inner annulus & 1.23 & 1.11 & 1.23 & 1.050 \\
\hline
\end{tabular}

Table 1: The best fit masks and the relative SNR for $\operatorname{Br} \alpha$ and $\mathrm{Kp}$ bands. With the exception of the outer annuli, there is very little difference in mask size between $\mathrm{Br}-\alpha$ and $\mathrm{Kp}$.

brighter at longer wavelengths. However, the uniform background does not seem to scale with wavelength as the thermal emitters do, as it is much more dominant in the shortest wavelengths than in the longest wavelengths.

\subsection{Pupil Mask Modeling}

We modeled the background-limited signal to noise ratio using hexagonal and annular-shaped masks of varying sizes. We separately tested inner masks, which block all emission within the annulus or hexagon and outer masks, which block all emission exterior of the hexagon or annulus. The outer hexagonal mask was oriented to align with the serrated primary mirror edge while the inner hexagonal mask was oriented such that each corner aligned with a secondary support, as shown in Figure 5b. We modeled a signal with our model of the primary mirror without the secondary obscuration, arbitrarily normalized. For each pupil mask, we nulled out anything inside or outside of the mask in the signal and noise image and summed the remaining flux. In Figure 4 we show the relative signal to noise of masks in $\mathrm{Kb}$ band $(2.1 \mu \mathrm{m})$ and $\mathrm{Br}-\alpha$ band $(4.1 \mu \mathrm{m})$. The radii for the best pupil masks are shown in Table 1 and are displayed in Figure 5.

For the inner component of the mask, the circular annulus had better signal to noise in all bands, despite the fact that the hexagonal mask covers some of the secondary mirror struts. The SNR as a function of radius, shown in Figure 4a is sharply peaked at the size of the secondary obscuration. The best pupil mask for the outer mask was also the annular mask. As shown in Figure 5, the annular mask, which was much more aggressive in background rejection. the difference between the two bands is most marked in this outer annular mask. As shown in Table 1, the pupil outer annular mask has a much smaller radius due to the higher large flux contribution from the dome and primary support structure.

\section{SUMMARY AND FUTURE WORK}

The goal of this project is to design pupil masks for the upcoming upgrade of the OSIRIS imager. In order to do so, we will need to take into account a number of factors not addressed here. We will have to account for the 


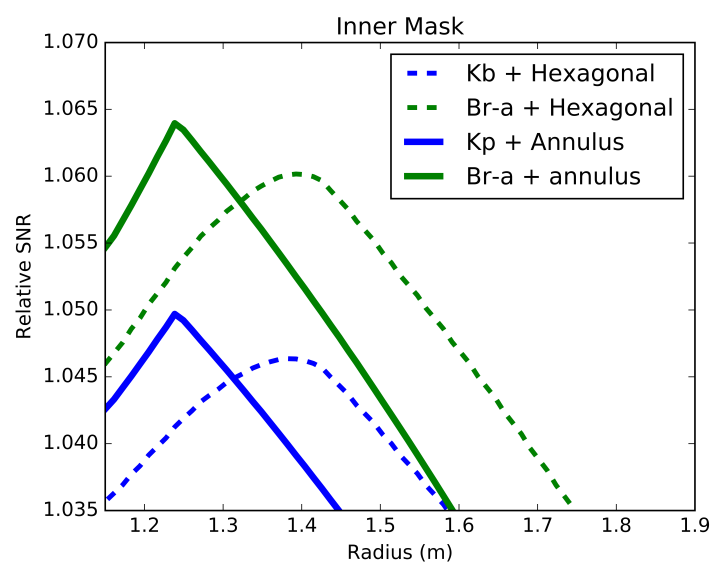

(a) Inner mask signal to noise

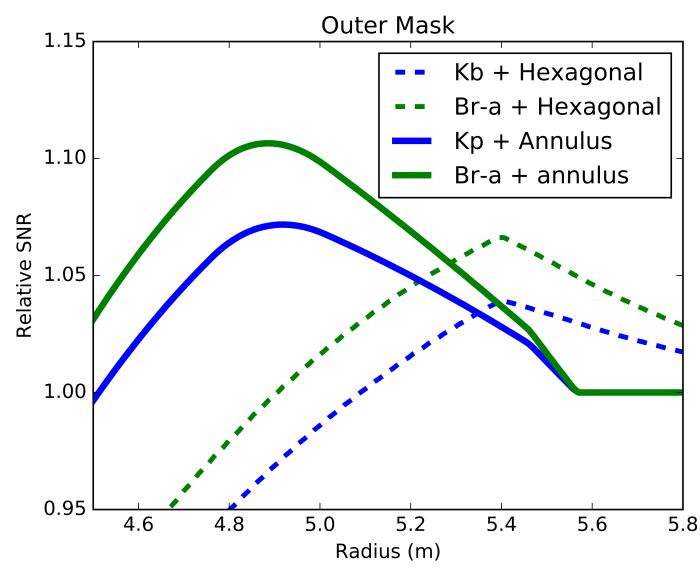

(b) Outer mask signal to noise

Figure 4: The relative signal to noise for $\mathrm{Kp}$ band (thicker blue lines) and $\mathrm{Br}-\alpha$ (thinner green lines) with hexagonal masks (dashed lines) and annular masks (solid lines). The radii shown are in units of meters in the primary plane.

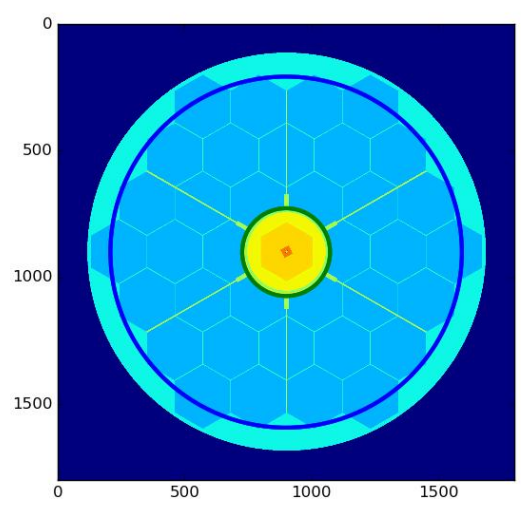

(a) Best fit circular masks.

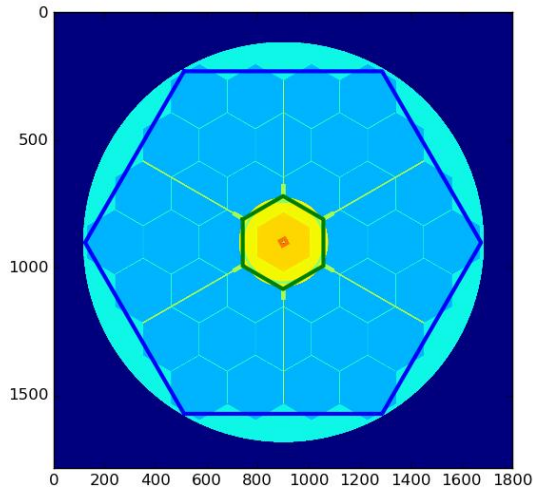

(b) Best fit annular masks.

Figure 5: The best fit inner masks for $\mathrm{Kp}$ in green and outer masks in blue. The sizes of these masks are shown in Table 1. 
nutation of the Keck pupil and error in pupil alignment. We will also have to account for constraints and limits in precision in manufacturing and aligning these pupil masks. These limiting factors will allow us to assess the feasibility of using a pupil mask that is matched to the serrated edge of the primary mirror.

Additionally, since we would like to have pupil masks for each filter, we would like to have models in every wavelength. This can be done by obtaining images in the other bands or by extrapolating our model into other wavelengths. In order to extrapolate our model, further calibrations will be needed in order to convert our flux parameters into physical units.

\section{ACKNOWLEDGMENTS}

The authors with to thank the generous support of the G. B. Moore Foundation (award 3847).

\section{REFERENCES}

Le Mignant, David, "Requirements for the Pupil Alignment on NIRC2." (1996) Keck Adaptive Optics Note 253. Nelson J., et al., "The design of the Keck observatory and telescope." (1985) Keck Observatory Report 90. 